Health, Faculty of Medicine, Norwegian University of Science and Technology; ${ }^{2}$ Department of Pediatrics, St. Olavs Hospital, Trondheim University Hospital, Trondheim, ${ }^{3}$ The Cerebral Palsy Registry of Norway, Habilitation Center, Vestfold Hospital, Tønsberg; ${ }^{4}$ National Center for Fetal Medicine, Department of Obstetrics and Gynecology, St. Olavs Hospital, Trondheim University Hospital, Trondheim; ${ }^{5}$ Medical Bith Registry of Norway, Locus of Registry-Based Epidemiological Research, Norwegian Institute of Public Health; ${ }^{6}$ Department of Public Health and Primary Health Care, University of Bergen, Bergen, Norway; 'National Perinatal Epidemiology Unit (NPEU), University of Oxford, Oxford, UK

Background and Aims To investigate the role of antenatal versus intrapartum causes in the pathway leading to cerebral palsy (CP) in children born small for gestational age (SGA) at term.

Methods Data on 400488 singleton term live births during 1996-2003 recorded in the Medical Birth Registry of Norway were linked with clinical data for 342 children diagnosed with CP recorded in the CP Registry of Norway. 'Low' Apgar score, defined as Apgar score $<4$ at five minutes, MRI-findings and subtype of CP were used to assess the timing of the brain injuries leading to CP.

Results In the group of 69 SGA children with CP, six (9\%; CI: 4-18) had 'low' Apgar scores, and five of these were considered to be of intrapartum origin (7\%; CI: 3-16). In the group of 263 non-SGA children with CP, 26 (10\%; CI: 7-14) had 'low' scores, and 18 of these probably had an intrapartum cause. In addition, an intrapartum cause was assessed as probable in 13 cases among children with Apgar scores $>3$. Thus, an intrapartum cause was considered likely in 31 non-SGA children (12\%; CI: 8-16), not different from the SGA group ( $\mathrm{p}=0.31)$.

Conclusions Despite increased odds of both low Apgar score and CP among children born SGA, our findings suggest that that the role of intrapartum causes in the causal chain leading to $\mathrm{CP}$ in these children is limited. Instead the results suggest that the majority of children with CP born SGA have antenatal brain injuries, also supported by MRI-findings.

\section{PROPHYLACTIC ANTIBIOTICS AND SEPSIS IN NEONATES BORN THROUGH MECONIUM STAINED AMNIOTIC FLUID (MSAF) - A RANDOMIZED CONTROLLED TRIAL}

doi:10.1136/archdischild-2012-302724.0391

${ }^{1} \mathrm{~A}$ Goel, ${ }^{2} \mathrm{~S}$ Nangia, ${ }^{2} \mathrm{~A}$ Saili, ${ }^{3} \mathrm{~S}$ Sharma, ${ }^{4} \mathrm{~V}$ Randhawa. 'Department of Pediatrics, ${ }^{2}$ Division of Neonatology, Department of Pediatrics, Lady Hardinge Medical College and Kalawati Saran Children's Hospital, New Delhi; ${ }^{3}$ Department of Pathology; ${ }^{4}$ Department of Microbiology, Lady Hardinge Medical College, New delhi, India

Background Most newborns with MSAF receive antibiotics as meconium has been incriminated to increase incidence of both intraamniotic and postnatal sepsis. Due to rising concerns about inadvertent overuse of antibiotics, this practice needs to be systematically evaluated.

Objective To evaluate the role of prophylactic antibiotics on occurrence of neonatal sepsis in term neonates born through MSAF. Methods Out of 359 eligible neonates, 109 were excluded based on exclusion criteria and remaining 250 randomized to Study (Antibiotic group - receiving first line antibiotics for 3 days), and Control (No Antibiotic) group. Both the groups were evaluated for sepsis on clinical and laboratory parameters. All neonates were monitored for complications related to MSAF. After discharge babies were followed up for sepsis till 28 days of life.

Results 121 babies were randomized to Antibiotic group and 129 to No Antibiotic group. Of the total 250 neonates, 24 (9.6\%) developed suspected sepsis, 8 in Antibiotic (6.6\%) and 16 in No Antibiotic group (12.4\%) ( $p=0.12$, OR 0.5, 95\% CI: 0.21-1.22). Culture proven sepsis occurred in 12 babies (4.8\%), 5 in Antibiotic and 7 in No Antibiotic group ( $4.13 \%$ vs. $5.42 \%, p=0.63$, OR $0.75,95 \%$ CI: $0.23-2.43$ ). The incidence of mortality ( $2.5 \%$ vs. $2.3 \%$ ), meconium aspiration syndrome $(18.2 \%$ vs. $15.5 \%, \mathrm{p}=0.57)$ and other complications like air leaks, PPHN and intracranial hemorrhage was comparable between the two groups.

Conclusions Prophylactic antibiotics in neonates born through MSAF do not reduce the incidence of sepsis. Hence, empiric use of antibiotics without documented evidence of infection should be avoided.

\section{PREVALENCE OF THE SYSTEMIC INFLAMMATORY RESPONSE SYNDROME, SEPSIS, SEVERE SEPSIS AND SEPTIC SHOCK IN A NEONATAL INTENSIVE CARE UNIT}

doi:10.1136/archdischild-2012-302724.0392

N Hofer, E Zacharias, W Mueller, B Resch. Medical University of Graz, Graz, Austria

Aim To examine the prevalence of the definitions of the systemic inflammatory response syndrome (SIRS), sepsis, severe sepsis, and septic shock during the first three days of life.

Methods Retrospective cohort study including all term neonates hospitalized at our neonatal intensive care unit within the first 24 hours of life from 2004 to 2010. SIRS and the different stages of sepsis were defined according to the International Pediatric Sepsis Consensus Conference.

Results 476 neonates included had a median birth weight of $3250 \mathrm{~g}$ (range 1250-5300g), a median gestational age of 38 weeks (37-43 weeks), and 258 (54\%) were male. Of 476 neonates included 116 (24\%) had SIRS, 61 (13\%) had sepsis, 55 (12\%) had severe sepsis, and $28(6 \%)$ had septic shock. Among 116 neonates with SIRS the single diagnostic criteria were fulfilled as follows: $37 / 116$ neonates (32\%) had fever or hypothermia, 92 (79\%) had a white blood cell count $>34000 / \mu \mathrm{l}$ and/or an immature to total neutrophil ratio $>0.1$, 115 (99\%) had respiratory and 40 (34\%) cardiocirculatory symptoms.

Conclusion A quarter of all term neonates hospitalized in our neonatal intensive care unit had SIRS during the first three days of life, half of them had sepsis. The vast majority of infants with sepsis had severe sepsis.

\section{HIGH-FLOW NASAL CANNULA VERSUS NASAL CONTINUOUS POSITIVE AIRWAY PRESSURE IN THE MANAGEMENT OF RESPIRATORY DISTRESS SYNDROME}

doi:10.1136/archdischild-2012-302724.0393

R Iranpour, A Sadeghnia, M Hesaraki. Pediatrics/Neonatology, Isfahan University of Medical Sciences, Isfahan, Iran

Background and Aims Although nasal continuous positive airway pressure (NCPAP) in the treatment of respiratory distress syndrome (RDS) is an effective and a non-invasive method, some complications such as septal trauma and intolerance of NCPAP apparatus are occurred. Our objectives are to assess safety and effectiveness of humidified high flow nasal canola (HFNC) as compared to NCPAP in premature neonates with RDS.

Methods Seventy uncomplicated preterm infant (30-35 weeks gestation) with RDS at the neonatal ward of Shahid-Beheshti hospital, Isfahan, Iran, randomized into two groups; Group1 (CPAP) received NCPAP from birth and continued till respiratory distress (RD) and oxygen $\left(\mathrm{O}_{2}\right)$ need improved and Group 2 (HFNC) received NCPAP for the first 24 hours after birth, then standard HFNC till $\mathrm{RD}$ and $\mathrm{O}_{2}$ need improved. Short outcomes and some long outcomes compared between two groups.

Results There were no differences in death, duration of hospitalization, failure to treatment, duration of improvement of $\mathrm{RD}$, necrotizing enterocolitis(NEC), patent ductus arteriosus (PDA), Intraventricular hemorrhage (IVH), chronic lung disease (CLD), pnumothorax, pulmonary hemorrhage, apnea, sepsis, duration of hospitalization, duration to reach to full entral feeding between 
two groups (NCPAP, HFNC). During the study, those on HFNC had more normal examination of nasal mucosa $(\mathrm{P}<0.0001)$. According to neonatal nurses opinions, application of HFNC was easier than NCPAP for neonates $(\mathrm{P}<0.0001)$.

Conclusions HFNC is as effective as NCPAP in the management of RDS in premature neonates more than 30 gestational weeks. In addition, HFNC performed easier than NCPAP with maintaining a normal nasal mucosa.

\section{EARLY DETECTION OF NEONATAL RESPIRATORY DECOMPENSATION LEADING TO INTUBATION USING PREDICTIVE MONITORING}

doi:10.1136/archdischild-2012-302724.0394

${ }^{1} \mathrm{MT}$ Clark, ${ }^{2} \mathrm{BD}$ Vergales, 'DE Lake, ${ }^{2} \mathrm{KD}$ Fairchild, ${ }^{2} \mathrm{AO}$ PagetBrown, ${ }^{2} \mathrm{~A}$ Bowe, ${ }^{2} \mathrm{~J}$ Kattwinkel, 'JR Moorman. 'Internal Medicine; ${ }^{2}$ Pediatrics, University of Virginia, Charlottesville, VA, USA

Aims Very low birth weight (VLBW $<1500$ grams) infants in the Neonatal Intensive Care Unit (NICU) are at risk for respiratory deterioration requiring endotracheal intubation and mechanical ventilation, with associated morbidities. Methods for predicting impending respiratory failure are needed, as timely non-invasive treatments might avert severe deterioration and the need to intubate.

Our aim was to develop a predictive statistical model for continuous analysis of cardiorespiratory waveforms and vital signs to predict respiratory failure requiring intubation in VLBW infants.

Methods We collected continuous cardiorespiratory and demographic data, and types and times of respiratory support on all VLBW infants admitted to the University of Virginia NICU from January 2009-June 2011. We identified non-elective intubations that were followed by mechanical ventilation for at least $12 \mathrm{~h}$. Over 25 physiological measures were tested, and a multivariate logistic regression model was developed to estimate the relative risk of urgent intubation in the next 24 hours.

Results Of 287 VLBW infants admitted, 96 urgent intubations in which there were at least $12 \mathrm{~h}$ of waveform data occurred in 51 patients. The final model had ROC area 0.84 and employed oxygen saturation and its cross-correlation with heart rate, cross-correlation of heart and respiratory rates, and apnea burden. Inspection showed rising risk of intubation over the 12 to 24 hours prior to the event. Conclusion Predictive monitoring of cardiorespiratory waveform patterns and vital signs can detect incipient respiratory failure as much as $24 \mathrm{~h}$ prior to urgent intubation.

\begin{tabular}{|l|l}
\hline 395 & NASAL INTERMITTENT MANDATORY VENTILATION \\
VERSUS NASAL CONTINUOUS POSITIVE AIRWAY \\
PRESSURE FOR TRANSIENT TACHYPNEA OF THE \\
NEWBORN: A RANDOMIZED PROSPECTIVE STUDY
\end{tabular}

doi:10.1136/archdischild-2012-302724.0395

'G Demirel, 'N Uras, 'IH Celik, 'FE Canpolat, 1,2U Dilmen. 'Neonatology, Zekai Tahir Burak Maternity and Teaching Hospital; ${ }^{2}$ Pediatrics, Yildirim Beyazıt University, Faculty of Medicine, Ankara, Turkey

Background and Aims Nasal continuous positive airway pressure (NCPAP) is widely used for the treatment of transient tachypnea of the newborn (TTN). In this study we hypothesized that the use of nasal intermittent mandatory ventilation (NIMV) may be well tolerated in TTN and we aimed to evaluate its efficacy in reducing the duration of respiratory distress compared with NCPAP in TTN.

Methods This prospective, unblinded, randomized, controlled clinical trial was conducted in 40 eligible infants with a gestational age $\geq 37$ weeks, and birth weight $\geq 2000 \mathrm{~g}$ who were hospitalized for TTN. Infants were randomized to either nonsynchronized NIMV $(n=20)$ or NCPAP $(n=20)$. The primary end point was the reduction of the duration of respiratory distress. Secondary end points were the duration and level of oxygen supplementation, the incidence of complications such as pneumothorax, pneumonia and respiratory failure requiring entubation.

Results There was no significant difference in the demographic features of the groups. There were no significant difference in the duration of respiratory support $(28.0 \pm 19.2 \mathrm{~h}$ vs $32.2 \pm 23.3 \mathrm{~h}$, $\mathrm{p}=0.231), \mathrm{O}_{2}$ therapy $(31.2 \pm 15.6 \mathrm{~h}$ vs $29.0 \pm 19.3 \mathrm{~h}, \mathrm{p}=0.187)$, duration of TTN $(67.6 \pm 36.5 \mathrm{~h}$ vs $63.3 \pm 39.1 \mathrm{~h}, \mathrm{p}=0.480)$ and hospitalization $(6.2 \pm 2.6 \mathrm{~d}$ vs $5.4 \pm 2.0 \mathrm{~d}, \mathrm{p}=0.330)$ between the groups. The rate of complications were not significantly different between the groups.

Conclusions Our study indicates that NIMV is well tolerated and as effective as NCPAP in the treatment of TTN.

\section{CURRENT PRACTICE REGARDING THE USE OF HUMIDIFIED HIGH FLOW NASAL CANNULAE (HHFNC) IN UK NEONATAL UNITS}

doi:10.1136/archdischild-2012-302724.0396

'P Desai, 'S Shetty, ${ }^{2} \mathrm{~N}$ Singh, ${ }^{1} \mathrm{~N}$ Kennea. ${ }^{1} 1{ }^{\text {St }}$ George's Hospital NHS Trust; ${ }^{2}$ Ealing Hospital NHS Trust, London, UK

Background Neonatal respiratory support using high flows of heated/humidified gas via nasal cannulae has gained acceptance in the UK despite limited evidence of efficacy and safety. HHFNC seems well tolerated with little reported airway trauma, reduced noise exposure, and easier nursing than NCPAP.

Aim To determine current practices in terms of usage, types of devices and weaning regimes of HHFNC.

Methods All 203 neonatal units across UK were contacted and a structured telephone questionnaire completed.

Results All 203 UK neonatal units (100\%) completed the survey, 113 neonatal units $(56 \%)$ use some form of HHFNC. There is more HHFNC use in level 3 neonatal intensive care units (Table 1).

Of the units using HHFNC, 47 (42\%) use HHFNC either as standard respiratory support following extubation or following NCPAP with the remainder (58\%) using NCPAP initially and then HHFNC. The majority $(60 \%)$ of units commenced HHFNC at 8 litres per minute (lpm) flow and $30 \%$ of units at $5-61 \mathrm{pm}$ and reduced in $0.5-11 \mathrm{pm}$ steps to wean. Most units weaned off HHFNC once the flow rate was $2-31 \mathrm{pm}$.

Conclusion This is a large UK study evaluating the use of HHFNC. This survey demonstrates increasing use of HHFNC and wide variations in methods of use and weaning parameters. There is a need for further research in this area.

Abstract 396 Table 1 Use of HHFNC according to Level of neonatal units

\begin{tabular}{llll}
\hline & $\begin{array}{l}\text { Special care baby } \\
\text { units (level 1) }\end{array}$ & $\begin{array}{l}\text { Local neonatal } \\
\text { units (level 2) }\end{array}$ & $\begin{array}{l}\text { Neonatal intensive } \\
\text { care units(level 3) }\end{array}$ \\
\hline HHFNC used (UK) & $12 / 53(23 \%)$ & $60 / 92(64 \%)$ & $41 / 58(70 \%)$ \\
HHFNC used \% England/ & $28 \% / 0 \% / 0 \% / 0 \%$ & $64 \% / 50 \% / 100 \% / 50 \%$ & $71 \% / 50 \% / 83 \% / 100 \%$ \\
$\begin{array}{l}\text { Wales/Scotland/Northern } \\
\text { Ireland }\end{array}$ & & & \\
\hline
\end{tabular}

397 HIGH FLOW NASAL CANNULAE CAUSE LESS NASAL TRAUMA COMPARED TO NASAL CONTINUOUS POSITIVE AIRWAY PRESSURE IN PRETERM INFANTS

doi:10.1136/archdischild-2012-302724.0397

C Collins. Neonatal Medicine, Mercy Hospital for Women, Melbourne, VIC, Australia

Background Nasal continuous positive airways pressure (NCPAP) facilitates extubation but can cause nasal trauma which ever device is used. The burden of nasal trauma is poorly defined in the literature. Nasal trauma often occurs in the smallest infants who require 